\title{
PBAR Note 656 \\ Chromaticity Measurements using Phase Modulated RF and Vector \\ Signal Analyzers
}

Dave McGinnis

February 16, 2001

\section{INTRODUCTION}

Chromaticity measurements are usually done by changing the energy of the beam by a known amount and measuring the change in betatron tune with a spectrum analyzer. The energy change is done by adjusting the RF frequency. The change in RF frequency is made large enough so that the change in betatron tune can be seen. If synchrotron motion is present in the beam, then measuring the change in betatron tune can be difficult.

This note will outline a method to measure the change in betatron tune by phasemodulating the RF and measuring the phase modulated betatron spectrum Extremely small resolution bandwidths are available on modern vector signal analyzers. A small resolution bandwidth is equivalent to measuring the chromaticity many times and averaging the results. This would permit much smaller shifts in betatron tunes to be measured. The phase-modulated signal consists of sidebands whose amplitudes are given by Bessel functions. The complication of the Bessel functions can be removed if the vector signal analyzer is capable of phase demodulation. The sign of the chromaticity can be determined by observing the modulation spectrum at both betatron sidebands.

\section{THEORY WITHOUT SYNCHROTRON MOTION}

This first part of this note will assume a single bunch undergoing betatron oscillations in a circular accelerator without any synchrotron motion. The azimuthal location of the bunch in the accelerator is defines as $\phi_{\mathbf{r}}$. In one turn around the machine, the ratio of betatron phase to azimuthal phase is defined as the betatron tune.

$$
\frac{\mathrm{d} \phi_{\beta}}{\mathrm{d} \phi_{\mathrm{r}}}=\mathrm{Q}
$$

The betatron tune has a nominal value of $\mathbf{Q}_{\mathbf{o}}$ on the central orbit. For particles with a different energy than the central orbit, the variation in tune from the nominal tune is given by the chromaticity:

$$
\mathrm{Q}=\mathrm{Q}_{\mathrm{o}}+\chi \frac{\Delta \mathrm{pc}}{\mathrm{pc}}
$$

The time dependence of the betatron phase averaged over one turn is given as:

$$
\mathrm{d} \phi_{\beta}=\mathrm{Q} \frac{\mathrm{d} \phi_{\mathrm{r}}}{\mathrm{dt}} \mathrm{dt}
$$

This note will assume that a phase shifter is placed downstream of the frequency source of the accelerator's RF system. The RF system operates at a harmonic number $\mathbf{h}$. The controller of the phase shifter will shift the RF phase at a frequency $\boldsymbol{\Omega}_{\text {mod }}$. The 
maximum amplitude of the phase shift is $\Delta \phi_{\mathbf{r f}}$. The time dependence of the bunch's azimuthal position is:

$$
\phi_{\mathrm{r}}=\omega_{\mathrm{r}} \mathrm{t}+\frac{\Delta \phi_{\mathrm{rf}}}{\mathrm{h}} \sin \left(\Omega_{\text {mod }} \mathrm{t}\right)
$$

The frequency at which the bunch travels around the accelerator is:

$$
\frac{\mathrm{d} \phi_{\mathrm{r}}}{\mathrm{dt}}=\omega_{\mathrm{r}}+\Omega_{\bmod } \frac{\Delta \phi_{\mathrm{rf}}}{\mathrm{h}} \cos \left(\Omega_{\bmod } \mathrm{t}\right)
$$

This note will assume that the revolution frequency of the central orbit is $\omega_{\mathrm{r}}$. The difference between the bunch's revolution frequency and the revolution frequency of the central orbit is:

$$
\Delta \omega_{\mathrm{r}}=\Omega_{\bmod } \frac{\Delta \phi_{\mathrm{rf}}}{\mathrm{h}} \cos \left(\Omega_{\bmod } \mathrm{t}\right)
$$

The energy difference between the bunch and the central orbit is given as:

$$
\begin{gathered}
\frac{\Delta \omega_{\mathrm{r}}}{\omega_{\mathrm{r}}}=-\eta \frac{\Delta \mathrm{pc}}{\mathrm{pc}} \\
\Delta \mathrm{pc}=-\frac{\mathrm{pc}}{\eta} \frac{\Omega_{\bmod }}{\omega_{\mathrm{r}}} \frac{\Delta \phi_{\mathrm{rf}}}{\mathrm{h}} \cos \left(\Omega_{\bmod } \mathrm{t}\right)
\end{gathered}
$$

Using Equations 2 and 3, the betatron frequency is:

$$
\begin{aligned}
\frac{\mathrm{d} \phi_{\beta}}{\mathrm{dt}} & =\omega_{\mathrm{r}} \mathrm{Q}_{\mathrm{o}}+\Omega_{\bmod } \frac{\Delta \phi_{\mathrm{rf}}}{\mathrm{h}}\left(\mathrm{Q}_{\mathrm{o}}-\frac{\chi}{\eta}\right) \cos \left(\Omega_{\text {mod }} \mathrm{t}\right) \\
& -\frac{\chi}{\eta \omega_{\mathrm{r}}}\left(\Omega_{\bmod } \frac{\Delta \phi_{\mathrm{rf}}}{\mathrm{h}}\right)^{2} \cos ^{2}\left(\Omega_{\text {mod }} \mathrm{t}\right)
\end{aligned}
$$

If:

$$
\frac{\Omega_{\bmod }}{\omega_{\mathrm{r}}} \frac{\Delta \phi_{\mathrm{rf}}}{\mathrm{h}}<<1
$$

then the second order term can be neglected. The betatron frequency becomes:

$$
\frac{\mathrm{d} \phi_{\beta}}{\mathrm{dt}}=\omega_{\mathrm{r}} \mathrm{Q}_{\mathrm{o}}+\Omega_{\mathrm{mod}} \frac{\Delta \phi_{\mathrm{rf}}}{\mathrm{h}}\left(\mathrm{Q}_{\mathrm{o}}-\frac{\chi}{\eta}\right) \cos \left(\Omega_{\text {mod }} \mathrm{t}\right)
$$

The betatron phase is:

$$
\phi_{\beta}=\omega_{\mathrm{r}} \mathrm{Q}_{\mathrm{o}} \mathrm{t}+\frac{\Delta \phi_{\mathrm{rf}}}{\mathrm{h}}\left(\mathrm{Q}_{\mathrm{o}}-\frac{\chi}{\eta}\right) \sin \left(\Omega_{\text {mod }} \mathrm{t}\right)+\phi_{\beta_{\mathrm{o}}}
$$

where $\phi_{\beta 0}$ is the betatron phase at $t=0$. The transverse position of the bunch at the pickup is: 


$$
\mathrm{x}_{\mathrm{pu}}=\sqrt{\beta_{\mathrm{pu}}} \mathrm{A} \cos \left(\phi_{\beta}\right)
$$

where $\mathbf{A}$ is the betatron amplitude of the bunch. If the bunch length is very small, the difference current available to the pickup is:

$$
\mathrm{I}_{\Delta}=\omega_{\mathrm{r}} \mathrm{q}_{\mathrm{b}} \frac{\mathrm{A}}{\sqrt{\varepsilon_{\max }}} \cos \left(\phi_{\beta}\right) \sum_{\mathrm{n}=-\infty}^{\infty} \delta\left(\phi_{\mathrm{r}}-2 \mathrm{n} \pi-\phi_{\mathrm{r}_{\mathrm{o}}}\right)
$$

where $\boldsymbol{\varepsilon}_{\mathbf{m a x}}$ is the aperture of the pickup, $\mathbf{q}_{\mathbf{b}}$ is the charge of the bunch, and $\phi_{\mathbf{r o}}$ is an arbitrary phase offset. The infinite sum of delta functions in Equation 14 can be expanded in a Fourier cosine series:

$$
\sum_{n=-\infty}^{\infty} \delta\left(\phi_{\mathrm{r}}-2 \mathrm{n} \pi-\phi_{\mathrm{r}_{\mathrm{o}}}\right)=\sum_{\mathrm{k}=1}^{\infty} \mathrm{C}_{\mathrm{k}} \cos \left(\mathrm{k}\left(\phi_{\mathrm{r}}-\phi_{\mathrm{r}_{\mathrm{o}}}\right)\right)
$$

where:

$$
\begin{aligned}
& \mathrm{C}_{0}=\frac{1}{2 \pi} \\
& \mathrm{C}_{\mathrm{k}}=\frac{1}{\pi}
\end{aligned}
$$

Using a simple trigonometric identity of the product of cosines, Equation 14 becomes:

$$
\begin{gathered}
\mathrm{I}_{\Delta}=\frac{\omega_{\mathrm{r}} \mathrm{q}_{\mathrm{b}}}{2} \frac{\mathrm{A}}{\sqrt{\varepsilon_{\max }}} \sum_{\mathrm{k}=0}^{\infty}\left(\mathrm{C}_{\mathrm{k}} \cos \left(\phi_{\mathrm{k}_{+}}\right)+\mathrm{C}_{\mathrm{k}} \cos \left(\phi_{\mathrm{k}_{-}}\right)\right) \\
\phi_{\mathrm{k}_{ \pm}}=\left(\mathrm{k} \pm \mathrm{Q}_{\mathrm{o}}\right) \omega_{\mathrm{r}} \mathrm{t}+\left(\mathrm{k} \pm \mathrm{Q}_{\mathrm{o}} \mp \frac{\chi}{\eta}\right) \frac{\Delta \phi_{\mathrm{rf}}}{\mathrm{h}} \sin \left(\Omega_{\text {mod }} \mathrm{t}\right)+\mathrm{k} \phi_{\mathrm{r}_{\mathrm{o}}} \pm \phi_{\beta_{\mathrm{o}}}
\end{gathered}
$$

where the + sign indicates the upper betatron sideband and the - sign indicates the lower betatron sideband. The frequency of the sidebands at revolution harmonic $\mathbf{k}$ is:

$$
\frac{\mathrm{d} \phi_{\mathrm{k}_{ \pm}}}{\mathrm{dt}}=\left(\mathrm{k} \pm \mathrm{Q}_{\mathrm{o}}\right) \omega_{\mathrm{r}}+\left(\mathrm{k} \pm \mathrm{Q}_{\mathrm{o}} \mp \frac{\chi}{\eta}\right) \frac{\Omega_{\mathrm{mod}} \Delta \phi_{\mathrm{rf}}}{\mathrm{h}} \cos \left(\Omega_{\mathrm{mod}} \mathrm{t}\right)
$$

Using the Bessel function relationship:

$$
\mathrm{e}^{\mathrm{jZ} \sin (\theta)}=\sum_{\mathrm{n}=-\infty}^{\infty} \mathrm{J}_{\mathrm{n}}(\mathrm{Z}) \mathrm{e}^{\mathrm{jn} \theta}
$$

Equation 17 becomes

$$
\begin{aligned}
\mathrm{I}_{\Delta} & =\frac{\omega_{\mathrm{r}} \mathrm{q}_{\mathrm{b}}}{2} \frac{\mathrm{A}}{\sqrt{\varepsilon_{\max }}} \sum_{\mathrm{k}=0}^{\infty} \mathrm{C}_{\mathrm{k}} \sum_{\mathrm{n}=-\infty}^{\infty} \mathrm{J}_{\mathrm{n}}\left(\mathrm{Z}_{+}\right) \cos \left(\omega_{\mathrm{k}, \mathrm{n}}^{+} \mathrm{t}+\psi_{+}\right) \\
& +\frac{\omega_{\mathrm{r}} \mathrm{q}_{\mathrm{b}}}{2} \frac{\mathrm{A}}{\sqrt{\varepsilon_{\max }}} \sum_{\mathrm{k}=0}^{\infty} \mathrm{C}_{\mathrm{k}} \sum_{\mathrm{n}=-\infty}^{\infty} \mathrm{J}_{\mathrm{n}}\left(\mathrm{Z}_{-}\right) \cos \left(\omega_{\mathrm{k}, \mathrm{n}}^{-} \mathrm{t}+\psi_{-}\right)
\end{aligned}
$$


where:

$$
\begin{gathered}
\mathrm{Z}_{ \pm}=\left(\mathrm{k} \pm \mathrm{Q}_{\mathrm{o}} \mp \frac{\chi}{\eta}\right) \frac{\Delta \phi_{\mathrm{rf}}}{\mathrm{h}} \\
\omega_{\mathrm{k}, \mathrm{n}}^{ \pm}=\left(\mathrm{k} \pm \mathrm{Q}_{\mathrm{o}}\right) \omega_{\mathrm{r}}+\mathrm{n} \Omega_{\mathrm{mod}} \\
\psi_{ \pm}=\mathrm{k} \phi_{\mathrm{r}_{\mathrm{o}}} \pm \phi_{\beta_{\mathrm{o}}}
\end{gathered}
$$

Equation 21 through Equation 24 show that the transverse spectrum will consist of a family of lines whose amplitude is a function of the chromaticity. One can determine the chromaticity by measuring the relative amplitude difference of the lines. The $\mathbf{J}_{\mathbf{1}}$ Bessel function and the $\mathbf{J}_{\mathbf{0}}$ Bessel functions are shown in Figure 1. By measuring the relative heights of the $\mathbf{J}_{\mathbf{0}}$ and $\mathbf{J}_{\mathbf{1}}$ lines at both betatron sidebands, the sign of the chromaticity can also be determined.

\section{THEORY WITH SYNCHROTRON MOTION}

The preceding section neglected the synchrotron motion of the bunch. If the bunch is also undergoing synchrotron oscillations as well as the phase modulation due to the RF system and the RF phase modulation frequency is much slower than the synchrotron frequency, then the azimuthal position of the particle is given as

$$
\phi_{\mathrm{r}}=\omega_{\mathrm{r}} \mathrm{t}+\frac{\Delta \phi_{\mathrm{rf}}}{\mathrm{h}} \sin \left(\Omega_{\bmod } \mathrm{t}\right)+\Delta \phi_{\mathrm{s}} \sin \left(\Omega_{\mathrm{s}} \mathrm{t}+\theta_{\mathrm{s}}\right)
$$

where $\Omega_{\mathrm{s}}$ is the synchrotron frequency and $\Delta \phi_{\mathrm{s}}$ is the synchrotron amplitude. If the RF and the synchrotron modulation is small, i.e.:

$$
\begin{gathered}
\frac{\Omega \bmod }{\omega_{\mathrm{r}}} \frac{\Delta \phi_{\mathrm{rf}}}{\mathrm{h}} \ll 1 \\
\frac{\Omega_{\mathrm{s}}}{\omega_{\mathrm{r}}} \Delta \phi_{\mathrm{s}} \ll 1
\end{gathered}
$$

Then the betatron phase can be written as:

$$
\phi_{\beta}=\omega_{\mathrm{r}} \mathrm{Q}_{\mathrm{o}} \mathrm{t}+\frac{\Delta \phi_{\mathrm{rf}}}{\mathrm{h}}\left(\mathrm{Q}_{\mathrm{o}}-\frac{\chi}{\eta}\right) \sin \left(\Omega_{\text {mod }} \mathrm{t}\right)+\Delta \phi_{\mathrm{s}}\left(\mathrm{Q}_{\mathrm{o}}-\frac{\chi}{\eta}\right) \sin \left(\Omega_{\mathrm{s}} \mathrm{t}+\theta_{\mathrm{s}}\right)+\phi_{\beta_{\mathrm{o}}}
$$

The frequency of the upper and lower betatron sidebands becomes 


$$
\begin{aligned}
\frac{\mathrm{d} \phi_{\mathrm{k}_{ \pm}}}{\mathrm{dt}} & =\left(\mathrm{k} \pm \mathrm{Q}_{\mathrm{o}}\right) \omega_{\mathrm{r}} \\
& +\left(\mathrm{k} \pm \mathrm{Q}_{\mathrm{o}} \mp \frac{\chi}{\eta}\right) \frac{\Omega_{\mathrm{mod}} \Delta \phi_{\mathrm{rf}}}{\mathrm{h}} \cos \left(\Omega_{\text {mod }} \mathrm{t}\right) \\
& +\left(\mathrm{k} \pm \mathrm{Q}_{\mathrm{o}} \mp \frac{\chi}{\eta}\right) \Omega_{\mathrm{s}} \Delta \phi_{\mathrm{s}} \cos \left(\Omega_{\mathrm{s}} \mathrm{t}\right)
\end{aligned}
$$

and the available difference mode current is:

$$
\begin{aligned}
\mathrm{I}_{\Delta} & =\frac{\omega_{\mathrm{r}} \mathrm{q}_{\mathrm{b}}}{2} \frac{\mathrm{A}}{\sqrt{\varepsilon_{\max }}} \sum_{\mathrm{k}=0}^{\infty} \mathrm{C}_{\mathrm{k}} \sum_{\mathrm{n}=-\infty}^{\infty} \sum_{\mathrm{m}=-\infty}^{\infty} \mathrm{J}_{\mathrm{m}}\left(\mathrm{Y}_{+}\right) \mathrm{J}_{\mathrm{n}}\left(\mathrm{Z}_{+}\right) \cos \left(\omega_{\mathrm{k}, \mathrm{m}, \mathrm{n}}^{+} \mathrm{t}+\psi_{+}\right) \\
& +\frac{\omega_{\mathrm{r}} \mathrm{q}_{\mathrm{b}}}{2} \frac{\mathrm{A}}{\sqrt{\varepsilon_{\max }}} \sum_{\mathrm{k}=0}^{\infty} \mathrm{C}_{\mathrm{k}} \sum_{\mathrm{n}=-\infty}^{\infty} \sum_{\mathrm{m}=-\infty}^{\infty} \mathrm{J}_{\mathrm{m}}\left(\mathrm{Y}_{-}\right) \mathrm{J}_{\mathrm{n}}\left(\mathrm{Z}_{-}\right) \cos \left(\omega_{\mathrm{k}, \mathrm{m}, \mathrm{n}}^{-} \mathrm{t}+\psi_{-}\right)
\end{aligned}
$$

where:

$$
\begin{gathered}
\mathrm{Y}_{ \pm}=\left(\mathrm{k} \pm \mathrm{Q}_{\mathrm{o}} \mp \frac{\chi}{\eta}\right) \Delta \phi \mathrm{s} \\
\omega_{\mathrm{k}, \mathrm{m}, \mathrm{n}}^{ \pm}=\left(\mathrm{k} \pm \mathrm{Q}_{\mathrm{o}}\right) \omega_{\mathrm{r}}+\mathrm{m} \Omega_{\mathrm{s}}+\mathrm{n} \Omega_{\bmod }
\end{gathered}
$$

Equations 30-32 show that every synchrotron sideband gets modulated with the RF phase modulation and that the chromaticity can be determined by examining the RF modulation lines around any synchrotron sideband.

\section{A TEVATRON EXAMPLE}

These numbers might be reasonable for the Tevatron at $150 \mathrm{GeV}$.

$$
\begin{gathered}
\chi=10 \\
\mathrm{~h}=1113 \\
\gamma_{\mathrm{t}}=18.8 \\
\gamma=160 \\
\mathrm{k}_{+}+\mathrm{Q}=1113 \times 21 \mathrm{MHz} / 53 \mathrm{MHz}=441 \\
\mathrm{k}-\mathrm{Q}=1113 \times 21 \mathrm{MHz} / 53 \mathrm{MHz}=441
\end{gathered}
$$

If $\phi_{\mathrm{rf}}=37$ degrees and $\Omega_{\bmod } / 2 \pi=20 \mathrm{~Hz}$ then:

$$
\begin{gathered}
\Delta \mathrm{p}_{\max } / \mathrm{p}=0.086 \times 10^{-3} \\
\mathrm{Z}_{+}=-1.8 \\
\mathrm{~J}_{0}(1.8)=0.34 \\
\mathrm{~J}_{1}(1.8)=0.582
\end{gathered}
$$




$$
\begin{gathered}
\mathrm{Z}_{-}=2.3 \\
\mathrm{~J}_{0}(2.3)=0.056 \\
\mathrm{~J}_{1}(2.3)=0.54
\end{gathered}
$$

Figure 2 shows a measured spectrum using an HP Vector Signal Analyzer of a $25 \mathrm{kHz}$ carrier signal that is phase modulated at $20 \mathrm{~Hz}$. The amplitude of the phase modulation was set at 1.75 radians (which would correspond to a $Z_{++}$of 1.75 ). A very nice feature of the HP vector signal analyzer is that it can demodulate the phase modulated signal and show the spectrum of the phase modulation which in this case would be a $20 \mathrm{~Hz}$ line with an amplitude of 1.75 radians (1.23 radians r.m.s). This spectrum is shown in Figure 3.

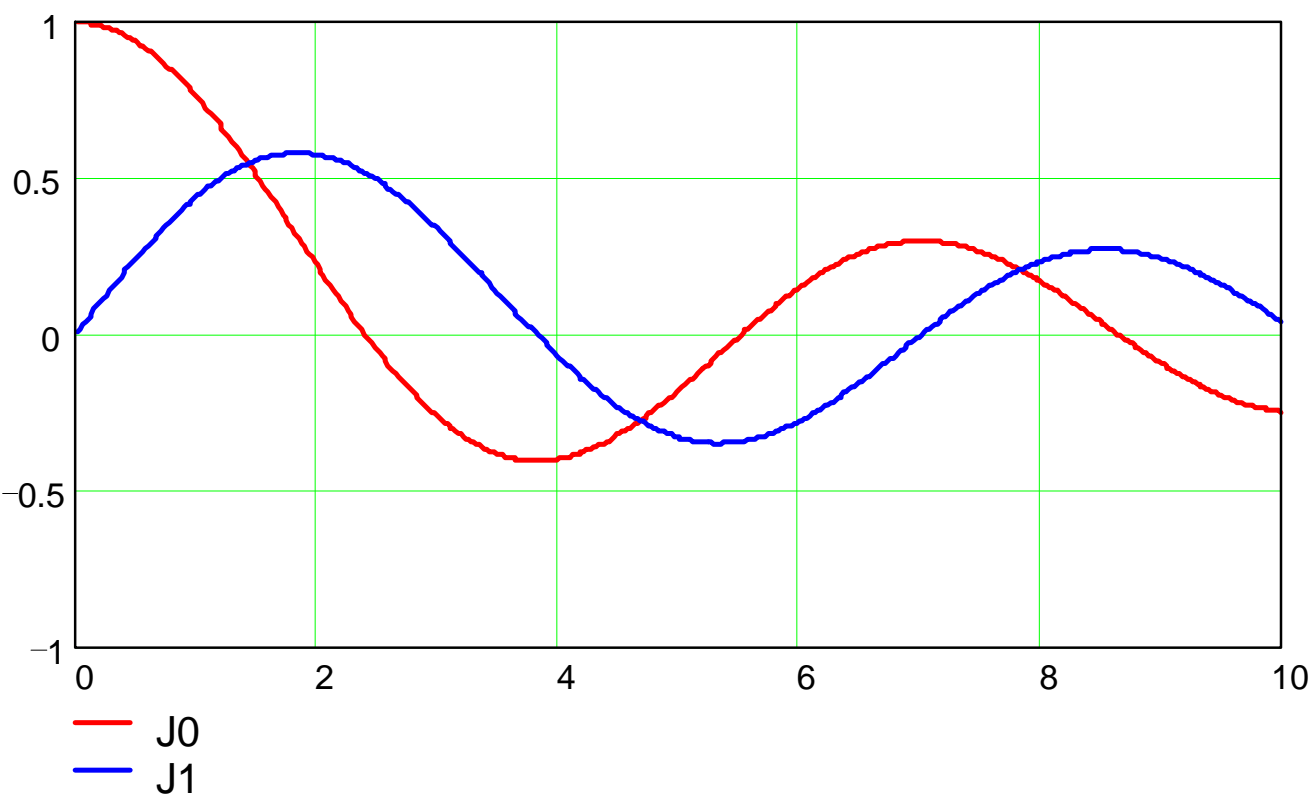

Figure 1. Bessel Functions 


\section{Date: 02-15-01 Time: 03:03 PM}

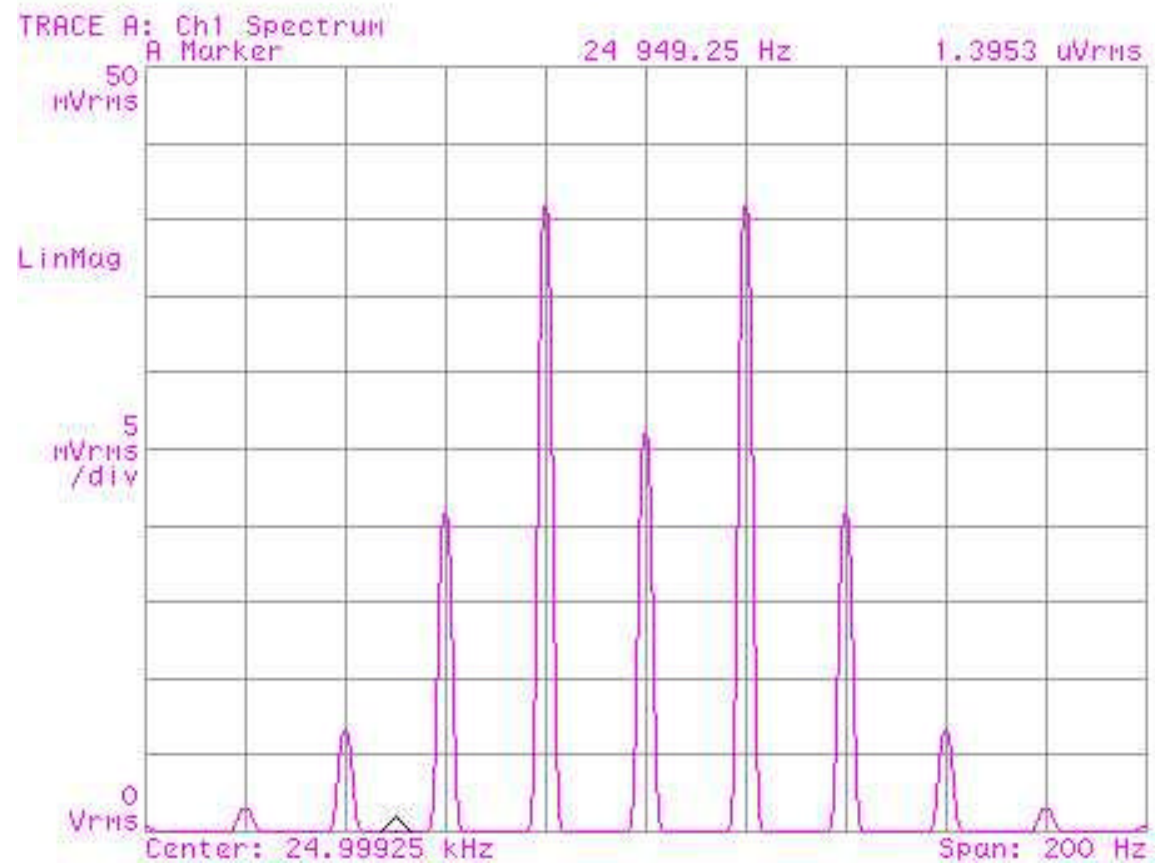

Figure 2. Spectrum of a phase modulated signal that has a $25 \mathrm{kHz}$ carrier modulated at rate of $20 \mathrm{~Hz}$ with a phase modulation amplitude of 1.75 radians

\section{Date: 02-15-01 Time: 02:50 PM}

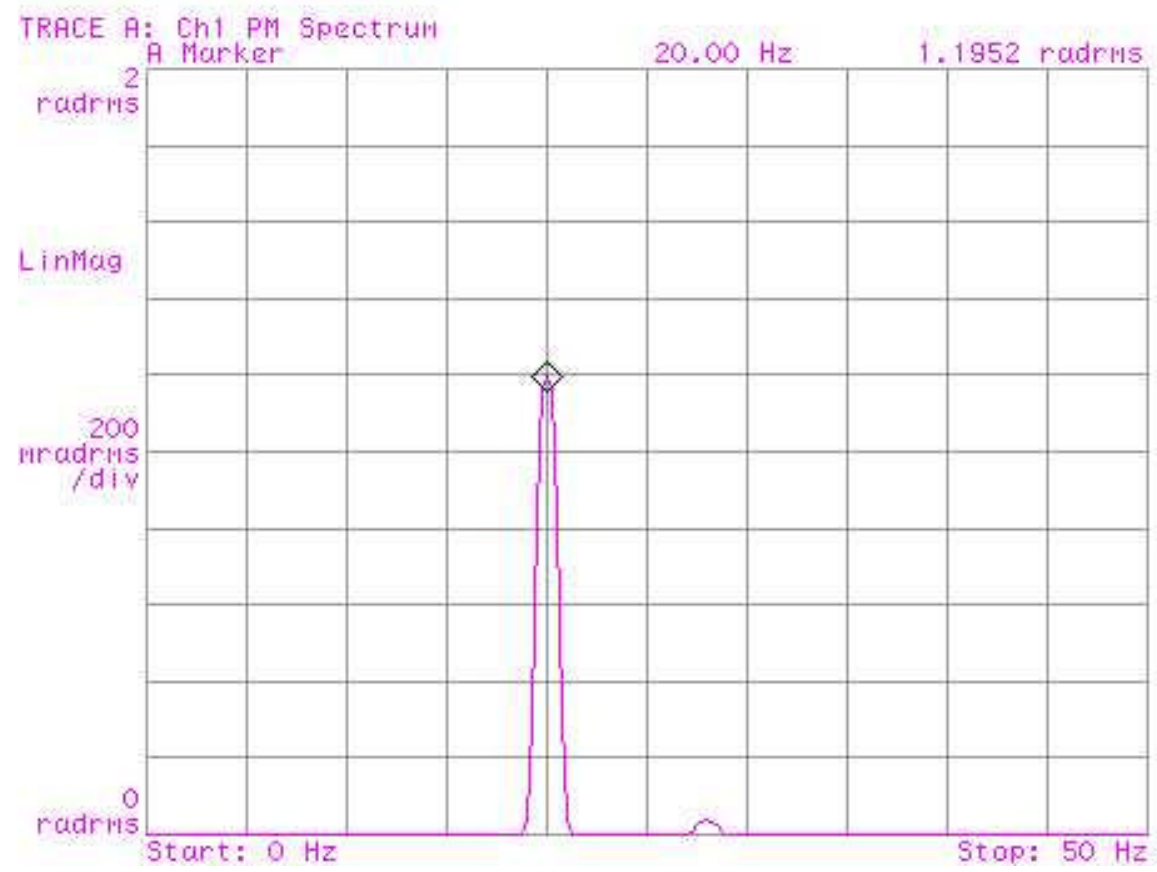

Figure 3. Demodulated spectrum of the spectrum shown in Figure 2. 